\title{
Flavors of Translative Coverings
}

\author{
Márton Naszódi
}

\begin{abstract}
We survey results on the problem of covering the space $\mathbb{R}^{n}$, or a convex body in it, by translates of a convex body. Our main goal is to present a diverse set of methods. A theorem of Rogers is a central result, according to which, for any convex body $K$, the space $\mathbb{R}^{n}$ can be covered by translates of $K$ with density around $n \ln n$. We outline four approaches to proving this result. Then, we discuss the illumination conjecture, decomposability of multiple coverings, Sudakov's inequality and some problems concerning coverings by sequences of sets.
\end{abstract}

2010 Mathematics Subject Classification $52 \mathrm{C} 17 \cdot 05 \mathrm{~B} 40 \cdot 52 \mathrm{~A} 23$

\section{Introduction}

The problem of covering a set by few translates of another appears naturally in several contexts. In computational applications it may be used for divide and conquer algorithms, in analysis, it yields $\varepsilon$-nets, in functional analysis, it is used to quantify how compact an operator between Banach spaces is. In geometry, it is simply an interesting question on its own.

Our primary focus is to describe a representative family of methods, rather than giving a complete account of the state of the art. In particular, we highlight some combinatorial ideas, and sketch some instructive probabilistic computations.

The author acknowledges the support of the János Bolyai Research Scholarship of the Hungarian Academy of Sciences, and the National Research, Development, and Innovation Office, NKFIH Grants PD104744 and K119670.

\author{
M. Naszódi (凶) \\ Department of Geometry, Lorand Eötvös University, Pázmány Péter Sétány 1/C, \\ Budapest 1117, Hungary \\ e-mail: marton.naszodi@math.elte.hu \\ (C) János Bolyai Mathematical Society and Springer-Verlag GmbH Germany, part of \\ Springer Nature 2018 \\ G. Ambrus et al. (eds.), New Trends in Intuitive Geometry, Bolyai Society \\ Mathematical Studies 27, https://doi.org/10.1007/978-3-662-57413-3_14
}


We minimize overlap with the fundamental works of L. Fejes Tóth [30] and Rogers [83]. Böröczky's book [18] is the most recent source on finite coverings. Some of the topics covered here are discussed in more detail in the books $[8,21,61,68]$. Many of the topics omitted, or only touched upon here (most notably, planar and three-dimensional results, lattice coverings and density) are discussed in the surveys [31, 32, 34-36].

In Sect. 3, we state Rogers' result, and a few of its relatives, on the existence of an economical covering of the whole space by translates of an arbitrary convex body. In Sect. 4, we outline three probabilistic proofs of these results. In Sect. 5, we describe a fourth approach, which is based on an algorithmic (non-probabilistic) result from combinatorics. Then, in Sect.6, we discuss the problem of illumination. There, we sketch the proof of a result of Schramm, which is currently the best general upper bound for Borsuk's problem. In Sect.7, we state some of the most recent results on the problem of decomposability of multiple coverings. Section 8 provides a window to how the asymptotic theory of convex bodies views translative coverings. Finally, in Sect. 9, we consider coverings by sequences of convex bodies.

We use the following notations, and terminology. For two Borel measurable sets $K$ and $L$ in $\mathbb{R}^{n}$, let $N(K, L)$ denote the translative covering number of $K$ by $L$, that is, the minimum number of translates of $L$ that cover $K$.

The Euclidean ball of radius one centered at the origin is $\mathbf{B}_{2}^{n}=\left\{x \in \mathbb{R}^{n}\right.$ : $\left.|x|^{2}=<x, x>\leq 1\right\}$, where $<., .>$ denotes the standard scalar product on $\mathbb{R}^{n}$. We denote the Haar probability measure on the sphere $\mathbb{S}^{n-1}=\left\{x \in \mathbb{R}^{n}:|x|=1\right\}$ by $\sigma$.

A symmetric convex body is a convex body (that is, a compact convex set with non-empty interior) that is centrally symmetric about some point. A hyperplane $H$ supports a convex set $K$, if $H$ intersects the boundary of $K$, and $K$ is contained in one of the closed half-spaces bounded by $H$. The support function $h_{K}$ of a convex set $K$ is defined as $h_{K}(x)=\sup \{<x, k>: k \in K\}$ for any $x \in \mathbb{R}^{n}$. We denote the polar of a convex body $K$ by

$$
K^{*}=\left\{x \in \mathbb{R}^{n}:<x, k>\leq 1 \text { for all } k \in K\right\} .
$$

The cardinality of a set $X$ is denoted by $|X|$.

\section{Basics}

We list a number of simple properties of covering numbers, their proofs are quite straight forward, cf. [3].

Fact 2.1 Let $K, L, M$ be convex sets in $\mathbb{R}^{n}, T: \mathbb{R}^{n} \rightarrow \mathbb{R}^{n}$ an invertible linear transformation. Then we have 


$$
\begin{aligned}
N(K, L) & =N(T(K), T(L)), \\
N(K, L) & \leq N(K, M) N(M, L), \\
N(K+M, L+M) & \leq N(K, L), \\
N(K, 2(K \cap L)) & \leq N(K, L), \text { if } K=-K .
\end{aligned}
$$

We note a special property of the Euclidean ball as a covering set.

Fact 2.2 Let $K$ be a convex set in $\mathbb{R}^{n}$. If $K$ is covered by $t$ Euclidean balls, then $K$ is covered by $t$ Euclidean balls with centers in $K$.

This fact follows from the observation that the intersection of $\mathbf{B}_{2}^{n}$ with a half-space not containing the origin is contained in the unit ball centered at the orthogonal projection of the origin to the bounding hyperplane of the half-space.

The following obvious lower bound is often sufficient:

$$
N(K, L) \geq \frac{\operatorname{vol}(L)}{\operatorname{vol}(K)}
$$

Next, we assume that $L$ is symmetric, and find an upper bound on $N(K, L)$. Let $X+(L / 2)$ be a saturated packing of translates of $L / 2$ in $K+(L / 2)$, that is a maximal family of translates of $L / 2$ with pairwise disjoint interiors. Then $K \subseteq$ $X+L$, that is, we have a covering of $K$ by $|X|$ translates of $L$. Thus,

$$
N(K, L) \leq|X| \leq 2^{n} \frac{\operatorname{vol}(K+(L / 2))}{\operatorname{vol}(L)} \text {, if } L=-L \text {. }
$$

Section 3, and a large part of this paper discuss how this bound can be improved.

\section{Covering the Whole Space}

Let $K$ be a convex body, $\Lambda$ a lattice, and $T$ a finite set in $\mathbb{R}^{n}$. We call the family $\mathcal{F}=$ $K+\Lambda+T=\{K+v+t: v \in \Lambda, t \in T\}$ a periodic arrangement of translates of $K$. The density of $\mathcal{F}$ is defined as $\delta(\mathcal{F})=|T| \operatorname{vol}(K) / \operatorname{det} \Lambda$. We say that $\mathcal{F}$ is a covering of $\mathbb{R}^{n}$ if $\cup \mathcal{F}=\mathbb{R}^{n}$. The translative covering density $\theta(K)$ of $K$ is the infimum of the densities of periodic coverings of $\mathbb{R}^{n}$ by translates of $K$. We note that one can define the density of a non-periodic arrangement, too (cf. [18, 30, 68, $83]$ ), and - as is easy to see - we obtain the same density infimum if we allow also non-periodic coverings of $\mathbb{R}^{n}$.

The first milestone in the theory of translative coverings is the following theorem of Rogers.

Theorem 3.1 (Rogers, [80]) Let $K$ be a bounded convex set in $\mathbb{R}^{n}$ with non-empty interior. Then the translative covering density of $K$ is at most 


$$
\theta(K) \leq n \ln n+n \ln \ln n+5 n
$$

Earlier, exponential upper bounds for the covering density were obtained by Rogers, Bambah and Roth, and for the special case of the Euclidean ball by Davenport and Watson (cf. [80] for references). The last summand, $5 n$ may be replaced by $3 n$, if $n$ is sufficiently large. The current best bound on $\theta(K)$ is due to G. Fejes Tóth [29], who replaced $5 n$ by $n+o(n)$ (see Theorem 3.4). It is an open problem whether one can improve the bound by a multiplicative factor below 1, or, very ambitiously, if $C n$ is an upper bound, for some universal $C>0$.

It is natural to ask what happens if the density is replaced by the maximum multiplicity.

Theorem 3.2 (Erdös, Rogers, [28]) For any convex body $K$ in $\mathbb{R}^{n}$ there is a periodic covering of $\mathbb{R}^{n}$ by translates of $K$ such that no point is covered by more than $e(n \ln n+n \ln \ln n+4 n)$ translates, and the density is below $n \ln n+n \ln \ln n+4 n$, provided $n$ is large enough.

A good candidate for a "bad" convex body, that is, one that cannot cover the space economically is the Euclidean ball, $\mathbf{B}_{2}^{n}$.

Theorem 3.3 (Coxeter, Few, Rogers, [22]) $\theta\left(\mathbf{B}_{2}^{n}\right) \geq C n$ with a universal constant $C>0$.

If we restrict ourselves to lattice coverings, that is, coverings of $\mathbb{R}^{n}$ by translates of a convex body $K$ where the translation vectors form a lattice in $\mathbb{R}^{n}$ (and denote the infimum of the densities by $\theta_{L}$ ), we have a much weaker bound. Rogers [82] (see also [83]) showed that for any convex body $K$, we have $\theta_{L}(K) \leq n^{\log _{2} \ln n+c}$. If $K$ has an affine image symmetric about at least $\log _{2} \ln n+4$ coordinate hyperplanes then, by a result of Gritzmann [41] (see also [68]), we have $\theta_{L}(K) \leq c n(\ln n)^{1+\log _{2} e}$. For the Euclidean ball, Rogers' estimate is the best known: $\theta_{L}\left(\mathbf{B}_{2}^{n}\right) \leq c n(\ln n)^{2.047}$.

The original proofs of Theorems 3.1 and 3.2 yield periodic coverings without any further structure. G. Fejes Tóth gave a proof of Theorem 3.1 that yields a covering with more of a lattice-like structure, and a slightly better density bound.

Theorem 3.4 (G. Fejes Tóth, [29]) For any convex body $K$ in $\mathbb{R}^{n}$ there is a lattice $\Lambda$ and a set $T \subset \mathbb{R}^{n}$ of $O(\ln n)$ translation vectors such that $K+\Lambda+T$ covers $\mathbb{R}^{n}$ with density at most $n \ln n+n \ln \ln n+n+o(n)$.

We give an outline of the proof of this result in Sect.4.3.

The following is a simple corollary to Theorem 3.1 (or the better bound, Theorem 3.4), which was first spelled out in [85].

Corollary 3.5 (Rogers and Zong [85]) Let $K$ and L be convex bodies in $\mathbb{R}^{n}$. Then

$$
N(K, L) \leq \frac{\operatorname{vol}(K-L)}{\operatorname{vol}(L)}(n \ln n+n \ln \ln n+n+o(n)) .
$$


Indeed, consider a covering $L+G$ of a large cube $C$ by translates of $L$ with density close to $n \ln n+n \ln \ln n+n+o(n)$. For any $t \in \mathbb{R}^{n}$, let $m(t)=\mid\{g \in G: K \cap$ $(g+t+L) \neq \emptyset\}|=| G \cap(K-L-t) \mid$. By averaging $m(t)$ over $t$ in $C$, we obtain that for some $t \in C$, we have $m(t) \leq \frac{\operatorname{vol}(K-L)}{\operatorname{vol}(L)}(n \ln \ln n+n \ln n+n+o(n)+\varepsilon)$.

In [24], Dumer showed that $\mathbb{R}^{n}$ can be covered with Euclidean unit balls of density around $\frac{1}{2} n \ln n$. A minor error in the proof was corrected in [25].

\section{Proofs of Theorems 3.1, 3.2 and 3.4}

\subsection{A Probabilistic Proof: Cover Randomly and then Mind the Gap}

We give an outline of Rogers' proof of Theorem 3.1.

We may assume that $K$ has volume one, and that the centroid (that is, the center of mass with respect to the Lebesgue measure) of $K$ is the origin. It follows that $K \subset-n K$. (Bonnesen and Fenchel in $\$ 34$. of [17] give several references to this fact: Minkowski [63] p. 105, Radon [79], Estermann [98] and Süss [94].)

Let $C$ be the cube $C=[0, R]^{n}$, where $R$ is large. Set $\eta=\frac{1}{n \ln n}$, and choose $N=$ $R^{n} n \ln \frac{1}{\eta}$ random translation vectors $x_{1}, \ldots, x_{N}$ in $C$ uniformly and independently. Let $\Lambda$ be the lattice $\Lambda=R \mathbb{Z}^{n}$. Thus, we obtain the family $K+\Lambda+\left\{x_{1}, \ldots, x_{N}\right\}$ of translates of $K$. The expected density of the union of this family is close to one, and hence, one can choose the $N$ translation vectors in such a way that the volume of the uncovered part of $C$ is small (at most $R^{n}\left(1-R^{-n}\right)^{N}$ ).

Next, we take a saturated (that is, maximal) packing $y_{1}-\frac{1}{n} K, \ldots y_{M}-\frac{1}{n} K$ of translates of $-\frac{1}{n} K$ inside this uncovered part of $C$. By the previous volume computation, we have few $\left(M \leq \eta^{-n} R^{n}\left(1-R^{-n}\right)^{N}\right)$ such translates. We replace each of these copies of $-\frac{1}{n} K$ by the same translate of $K$, make it a periodic arrangement by $\Lambda$, and we obtain $K+\Lambda+\left\{y_{1}, \ldots, y_{M}\right\}$.

Now, we have two families of translates of $K$. We enlarge each member of these two families by a factor $1+\eta$, and -as it is easy to see- obtain a covering of $\mathbb{R}^{n}$. The omitted computations yield the density bound, finishing the proof of Theorem 3.1.

This method (first, picking random copies, and then, filling the gap, which is small, in a greedy way), developed by Rogers can be applied for obtaining upper bounds in other situations as well. The proof of Theorem 3.2 given by Erdös and Rogers is an example of the use of this random covering technique combined with a sophisticated way of keeping track of multiply covered points using an inclusionexclusion formula. Other examples include bounds on covering the sphere $\mathbb{S}^{n-1}$ with spherical caps. 


\subsection{Another Probabilistic Proof: Using the Lovász Local Lemma}

Füredi and Kang [39] gave a proof of Theorem 3.2 that is essentially different from the original. Their method yields a slightly worse bound (instead of the order $e n \ln n$, they obtain $10 n \ln n$ ), but it is very elegant.

First, by considering an affine image of $K$, we may assume that $\operatorname{vol}(K)=1$, and $\frac{1}{e} \mathbf{B}_{2}^{n} \subset K$ (cf. [6], see also [7] for the symmetric case). Let $h=1 /(4 e n \sqrt{n})$, and consider the lattice $\Lambda=h \mathbb{Z}^{n}$. The goal is to cover $\mathbb{R}^{n}$ with translates of $K$ of the form $K+z$ with $z \in \Lambda$. Let $Q=[0, h)^{n}$ be the half closed, half open fundamental cube of $\Lambda$. We define a hypergraph with base set $\Lambda$. The hypergraph has two types of edges. For any $z \in \Lambda$, we define a "small edge" as $A^{-}(z):=\{y \in \Lambda: y+Q \subset z+K\}$, and a "big edge" as $A^{+}(z):=\{y \in \Lambda:(y+Q) \cap(z+K) \neq \emptyset\}$. Clearly, all big edges are of the same size (say $\alpha$ ), and so are all small edges. One can verify that the size of a small edge is at least $\alpha / 2$.

Next, to make the problem finite, let $\ell \in \mathbb{Z}^{+}$be an arbitrarily large integer. Our goal is to select vectors $z_{1}, \ldots, z_{t} \in \Lambda$ in such a way that every point of $[-\ell, \ell]^{n} \cap \Lambda$ is covered by a small edge $A^{-}\left(z_{i}\right)$, and no point of $[-\ell, \ell]^{n} \cap \Lambda$ is covered by more than $10 n \ln n$ large edges of the form $A^{+}\left(z_{i}\right)$. Clearly, that would suffice for proving the theorem. We will pick these vectors randomly: select each vector in $\left\{z \in \Lambda: A^{-}(z) \cap[-\ell, \ell]^{n} \neq \emptyset\right\}$ with probability $p$, where $p=e^{-6 / 5} 10 n \ln n / \alpha$.

For every point of $[-\ell, \ell]^{n} \cap \Lambda$, we have two kinds of bad events. One is if it is not covered by a small edge, and second, if it is covered by too many big edges. Now, we state the main tool of the proof, the Lovász Local Lemma (see Alon and Spencer [1] for a good introduction of it).

Lemma 4.1 (Lovász Local Lemma, $[27,91])$ Let $A_{1}, A_{2}, \ldots, A_{N}$ be events in an arbitrary probability space. A directed graph $D=(V, E)$ on the set of vertices $V=$ $\{1,2, \ldots, N\}$ is called a dependency digraph for the events $A_{1}, A_{2}, \ldots, A_{N}$, if for each $1 \leq i \leq N$, the event $A_{i}$ is mutually independent of all the events $\left\{A_{j}:(i, j) \notin\right.$ $E\}$. Suppose that the maximum degree of $D$ is at most $d$, and that the probability of each $A_{i}$ is at most $p$. If ep $(d+1) \leq 1$, then with positive probability no $A_{i}$ holds.

Finally, with a geometric argument, one can bound the maximum degree in a dependency digraph of the bad events, and Lemma 4.1 yields the existence of a good covering.

\subsection{Covering Using Few Lattices}

G. Fejes Tóth's proof of Theorem 3.4 relies on a deep result, Theorem 10* in [86] of Schmidt. A consequence of this result is

Lemma 4.2 Let $c_{0}=0.278 \ldots$ be the root of the equation $1+x+\ln x=0$. Then, for any $0<c<c_{0}$, and $\varepsilon>0$, and any sufficiently large $n$, for any Borel set $S \subset \mathbb{R}^{n}$, 
there is a lattice-arrangement of $S$ with density cn covering $\mathbb{R}^{n}$ with the exception of a set whose density is at most $(1+\varepsilon) e^{-c n}$ for some universal constant $c>0$.

By this lemma, for a given $K$ there is a lattice $\Lambda$ such that $\left(1+\lfloor n \ln n\rfloor^{-1}\right)^{-1} K+$ $\Lambda$ covers $\mathbb{R}^{n}$ with the exception of a set whose density is at most $e^{-c n+1}$.

Lemma 4.3 If, for some finite set $T, K+\Lambda+T$ is an arrangement of $K$ with density $1-\delta$, then there is a vector $t \in \mathbb{R}^{n}$ such that the arrangement $K+\Lambda+T^{\prime}$ has density at least $1-\delta^{2}$, where $T^{\prime}=T \cup(T+t)$.

The proof of Lemma 4.3 relies on considering the density of $\mathbb{R}^{n} \backslash\left(K+\Lambda+T^{\prime}\right)$ as a function of $t$, and averaging it over the fundamental domain of $\Lambda$.

To prove Theorem 3.4, we pick an appropriate $c$ for Lemma 4.2, and using Lemma 4.3 roughly $\log _{2}\left(c^{-1} \ln n\right)$ times, we obtain a finite set $T$ of size about $c^{-1} \ln n$ such that $\left(1+\lfloor n \ln n\rfloor^{-1}\right)^{-1} K+\Lambda+T$ has density about $n \ln n$ with the uncovered part being of density at most of order $(n \ln n)^{-n}$. Finally, one can verify that $K+\Lambda+T$ is a covering of space with the desired density.

So far, we presented three probabilistic methods that yield economical coverings. In the next section, we present a fourth method, which is not random. Instead, it relies on an algorithmic combinatorial result.

\section{A Fractional Approach}

\subsection{A Few Words of Combinatorics}

We recall some notions from the theory of hypergraphs.

Definition 5.1 Let $\Lambda$ be a set, $\mathcal{H}$ a family of subsets of $\Lambda$. A covering of $\Lambda$ by $\mathcal{H}$ is a subset of $\mathcal{H}$ whose union is $\Lambda$. The covering number $\tau(\Lambda, \mathcal{H})$ of $\Lambda$ by $\mathcal{H}$ is the minimum cardinality of its coverings by $\mathcal{H}$.

A fractional covering of $\Lambda$ by $\mathcal{H}$ is a measure $\mu$ on $\mathcal{H}$ with

$$
\mu(\{H \in \mathcal{H}: p \in H\}) \geq 1 \text { for all } p \in \Lambda \text {. }
$$

The fractional covering number of $\mathcal{H}$ is

$$
\tau^{*}(\Lambda, \mathcal{H})=\inf \{\mu(\mathcal{H}): \mu \text { is a fractional covering of } \Lambda \text { by } \mathcal{H}\}
$$

When $\Lambda$ is a finite set, finding the value of $\tau(\Lambda, \mathcal{H})$ is an integer programming problem. Indeed, we assign a variable $x_{H}$ to each member $H$ of $\mathcal{H}$, and set $x_{H}$ to 1 if $H$ is in the covering, and 0 otherwise. Each element $p$ of $\Lambda$ yields an inequality: $\sum_{p \in H \in \mathcal{H}} x_{H} \geq 1$. 
Computing $\tau^{*}(\Lambda, \mathcal{H})$ is the linear relaxation of the above integer programming problem. For more on (fractional) coverings, cf. [38] in the abstract (combinatorial) setting and $[61,68]$ in the geometric setting.

The gap between $\tau$ and $\tau^{*}$ is bounded in the case of finite set families (hypergraphs) by the following result.

Lemma 5.2 (Lovász [57], Stein [92]) For any finite $\Lambda$ and $\mathcal{H} \subseteq 2^{\Lambda}$ we have

$$
\tau(\Lambda, \mathcal{H})<\left(1+\ln \left(\max _{H \in \mathcal{H}}|H|\right)\right) \tau^{*}(\Lambda, \mathcal{H})
$$

Furthermore, the greedy algorithm (always picking the set that covers the largest number of uncovered points) yields a covering of cardinality less than the right hand side in (9).

We note that a probabilistic argument yields a slightly different bound on the covering number:

$$
\tau(\Lambda, \mathcal{H}) \leq\left\lfloor 1+\frac{\ln |\Lambda|}{-\ln \left(1-\frac{1}{\tau^{*}}\right)}\right\rfloor
$$

with the notation $\tau^{*}=\tau^{*}(\Lambda, \mathcal{H})$. When we do not have an upper bound on $\max _{H \in \mathcal{H}}|H|$ better than $|\Lambda|$, then (10) is a bit better than (9).

To prove (10), let $\mu$ be a fractional covering of $\Lambda$ by $\mathcal{H}$ such that $\mu(\mathcal{H})=\tau^{*}+\varepsilon$, where $\varepsilon>0$ is very small. We normalize $\mu$ to obtain the probability measure $\nu=$ $\mu / \mu(\mathcal{H})$ on $\mathcal{H}$. Let $m$ denote the right hand side in (10), and pick $m$ members of $\mathcal{H}$ randomly according to $\nu$. Then we have

$$
\mathbb{P}(\exists u \in \Lambda: u \text { is not covered }) \leq|\Lambda|\left(1-\frac{1}{\tau^{*}+\varepsilon}\right)^{m}<1 .
$$

Thus, with positive probability, we have a covering.

We will need the duals of these notions as well. Let $\Lambda$ be a set and $\mathcal{H}$ be a family of subsets of $\Lambda$. The dual of this set family is another set family, whose base set is $\mathcal{H}$, and the set family on $\mathcal{H}$ is $\mathcal{H}^{*}=\{\{H \in \mathcal{H}: p \in H\}: p \in \Lambda\}$.

We call a set $T \subset \Lambda$ a transversal to the set family $\mathcal{H}$, if $T$ intersects each member of $\mathcal{H}$. One may define fractional transversals in the obvious way, and then define the (fractional) transversal number.

Clearly $\mathcal{G} \subset \mathcal{H}$ is a covering of $\Lambda$ if and only if, $\mathcal{G}$ is a transversal to $\mathcal{H}^{*}$. Fractional coverings and fractional transversals are dual notions in the same manner. We leave it as an exercise (which will be needed later) to formulate the dual of Lemma 5.2 and of (10). 


\subsection{The Fractional Covering Number}

Motivated by the above combinatorial notions, the fractional version of $N(K$, int $K)$ (which is the illuminaton number of $K$, see Sect.6) first appeared in [65], and in general for $N(K, L)$ in [4] and [5].

Definition 5.3 Let $K$ and $L$ be bounded Borel measurable sets in $\mathbb{R}^{n}$. A fractional covering of $K$ by translates of $L$ is a Borel measure $\mu$ on $\mathbb{R}^{n}$ with $\mu(x-L) \geq 1$ for all $x \in K$. The fractional covering number of $K$ by translates of $L$ is

$$
\begin{gathered}
\qquad N^{*}(K, L)= \\
\inf \left\{\mu\left(\mathbb{R}^{n}\right): \mu \text { is a fractional covering of } K \text { by translates of } L\right\} .
\end{gathered}
$$

Clearly,

$$
N^{*}(K, L) \leq N(K, L)
$$

In Definition 5.3 we may assume that a fractional cover $\mu$ is supported on $\operatorname{cl}(K-$ $L)$. According to Theorem 1.7 of [5], we have

$$
\max \left\{\frac{\operatorname{vol}(K)}{\operatorname{vol}(L)}, 1\right\} \leq N^{*}(K, L) \leq \frac{\operatorname{vol}(K-L)}{\operatorname{vol}(L)}
$$

The second inequality is easy to see: the Lebesgue measure restricted to $K-L$ with the following scaling $\mu=\operatorname{vol} / \operatorname{vol}(L)$ is a fractional covering of $K$ by translates of $L$. To prove the first inequality, assume that $\mu$ is a fractional covering of $K$ by translates of $L$. Then

$$
\begin{gathered}
\operatorname{vol}(L) \mu\left(\mathbb{R}^{n}\right)=\int_{\mathbb{R}^{n}} \operatorname{vol}(L) \mathrm{d} \mu(x)=\int_{\mathbb{R}^{n}} \int_{\mathbb{R}^{n}} \chi_{L}(y-x) \mathrm{d} y \mathrm{~d} \mu(x)= \\
\int_{\mathbb{R}^{n}} \int_{\mathbb{R}^{n}} \chi_{L}(y-x) \mathrm{d} \mu(x) \mathrm{d} y=\int_{\mathbb{R}^{n}} \mu(y-L) \mathrm{d} y \geq \int_{\mathbb{R}^{n}} \chi_{K}(y) \mathrm{d} y=\operatorname{vol}(K) .
\end{gathered}
$$

We recall from Sect. 5.1 that computing $N$ means solving an integer programming problem (though, in this situation, with infinitely many variables), and computing $N^{*}$ is its linear relaxation. The linear relaxation is usually easier to solve, so having an inequality bounding $N$ from above by some function of $N^{*}$ is desirable. It is open whether such inequality exists in general for convex sets. More precisely, we do not know if there is a function $f$ such that for any dimension $n$, and any convex bodies $K$ and $L$ in $\mathbb{R}^{n}$, we have $N(K, L) \leq f\left(n, N^{*}(K, L)\right)$.

Using a probabilistic argument, Artstein-Avidan and Slomka [5] found a bound of $N(K, L)$ in terms of $N^{*}\left(K^{\prime}, L^{\prime}\right)$, where $K^{\prime}$ and $L^{\prime}$ are very close (but not identical) to $K$ and $L$. A somewhat stronger bound was obtained in [78] by a non-probabilistic 
proof. For two sets $K, T \subset \mathbb{R}^{n}$, we denote their Minkowski difference by $K \sim T=$ $\left\{x \in \mathbb{R}^{n}: T+x \subseteq K\right\}$.

Theorem 5.4 (Artstein-Avidan and Slomka [5], Naszódi [78]) Let $K, L$ and $T$ be bounded Borel measurable sets in $\mathbb{R}^{n}$ and let $\Lambda \subset \mathbb{R}^{n}$ be a finite set with $K \subseteq \Lambda+T$. Then

$$
\begin{gathered}
N(K, L) \leq \\
\left(1+\ln \left(\max _{x \in K-L}|(x+(L \sim T)) \cap \Lambda|\right)\right) \cdot N^{*}(K-T, L \sim T) .
\end{gathered}
$$

If $\Lambda \subset K$, then we have

$$
\begin{gathered}
N(K, L) \leq \\
\left(1+\ln \left(\max _{x \in K-L}|(x+(L \sim T)) \cap \Lambda|\right)\right) \cdot N^{*}(K, L \sim T) .
\end{gathered}
$$

We sketch a proof of Theorem 5.4 in 5.3.

For a set $K \subset \mathbb{R}^{n}$ and $\delta>0$, we denote the $\delta$-inner parallel body of $K$ by $K_{-\delta}:=K \sim \delta \mathbf{B}_{2}^{n}=\left\{x \in K: x+\delta \mathbf{B}_{2}^{n} \subseteq K\right\}$. As an application of Theorem 5.4, one quickly obtains the following result which, in turn, may be used to give a simple proof of Rogers' result, Theorem 3.1.

Theorem 5.5 (Naszódi [78]) Let $K \subseteq \mathbb{R}^{n}$ be a bounded measurable set. Then there is a covering of $\mathbb{R}^{n}$ by translated copies of $K$ of density at most

$$
\inf _{\delta>0}\left[\frac{\operatorname{vol}(K)}{\operatorname{vol}\left(K_{-\delta}\right)}\left(1+\ln \frac{\operatorname{vol}\left(K_{-\delta / 2}\right)}{\operatorname{vol}\left(\frac{\delta}{2} \mathbf{B}_{2}^{n}\right)}\right)\right] .
$$

A similar theorem holds if, in the definition of the $\delta$-inner parallel body, the Euclidean ball is replaced by some other convex body.

\subsection{Proof of Theorem 5.4}

The proofs outlined so far were all probabilistic in nature. In this one, the role that probability plays elsewhere is played by the following straightforward corollary to Lemma 5.2.

Observation 5.6 Let $Y$ be a set, $\mathcal{F}$ a family of subsets of $Y$, and $X \subseteq Y$. Let $\Lambda$ be a finite subset of $Y$ and $\Lambda \subseteq U \subseteq Y$. Assume that for another family $\mathcal{F}^{\prime}$ of subsets of $Y$ we have $\tau(X, \mathcal{F}) \leq \tau\left(\Lambda, \mathcal{F}^{\prime}\right)$. Then

$$
\tau(X, \mathcal{F}) \leq \tau\left(\Lambda, \mathcal{F}^{\prime}\right) \leq\left(1+\ln \left(\max _{F^{\prime} \in \mathcal{F}^{\prime}}\left|\Lambda \cap F^{\prime}\right|\right)\right) \cdot \tau^{*}\left(U, \mathcal{F}^{\prime}\right)
$$


The proof is simply a substitution into (15). We set $Y=\mathbb{R}^{n}, X=K, \mathcal{F}=\{L+$ $x: x \in K-L\}, \mathcal{F}^{\prime}=\{L \sim T+x: x \in K-L\}$. One can use $U=K-T$, as any member of $\Lambda$ not in $K-T$ could be dropped from $\Lambda$ and $\Lambda$ would still have the property that $\Lambda+T \supseteq K$. That proves (13). To prove (14), we notice that in the case when $\Lambda \subset K$, one can take $U=K$.

\subsection{Detour: Covering the Sphere by Caps}

To illustrate the applicability of the method that yields Theorem 5.4, we turn to coverings on the sphere. We denote the closed spherical cap of spherical radius $\phi$ centered at $u \in \mathbb{S}^{n-1}$ by $C(u, \phi)=\left\{v \in \mathbb{S}^{n-1}:\langle u, v>\geq \cos \phi\}\right.$, and its probability measure by $\Omega(\phi)=\sigma(C(u, \phi))$. For a set $K \subset \mathbb{S}^{n-1}$ and $\delta>0$, we denote the $\delta$-inner parallel body of $K$ by $K_{-\delta}=\{u \in K: C(u, \delta) \subseteq K\}$.

A set $K \subset \mathbb{S}^{n-1}$ is called spherically convex, if it is contained in an open hemisphere and for any two of its points, it contains the shorter great circular arc connecting them.

The spherical circumradius of a subset of an open hemisphere of $\mathbb{S}^{n-1}$ is the spherical radius of the smallest spherical cap (the circum-cap) that contains the set. A proof mimicking the proof of Theorem 5.4 yields

Theorem 5.7 (Naszódi [78]) Let $K \subseteq \mathbb{S}^{n-1}$ be a measurable set. Then there is a covering of $\mathbb{S}^{n-1}$ by rotated copies of $K$ of density at most

$$
\inf _{\delta>0}\left[\frac{\sigma(K)}{\sigma\left(K_{-\delta}\right)}\left(1+\ln \frac{\sigma\left(K_{-\delta / 2}\right)}{\Omega\left(\frac{\delta}{2}\right)}\right)\right] .
$$

Improving an earlier result of Rogers [81], Böröczky and Wintsche [19] showed that for any $0<\varphi<\pi / 2$ and dimension $n$ there is a covering of $\mathbb{S}^{n}$ by spherical caps of radius $\phi$ with density at most $n \ln n+n \ln \ln n+5 n$. This result follows from Theorem 5.7. Other bounds on covering the sphere by caps (or, a ball by smaller equal balls) can be found in [100] by Verger-Gaugry.

\section{The Illumination Conjecture}

We fix a convex body $K$ in $\mathbb{R}^{n}$. Once the covering number is defined, it is fairly natural to ask what Levi [56] asked: how large may $N(K$, int $K)$ be. We will call this quantity the illumination number of $K$, and denote it by $i(K)=N(K$, int $K)$. The naming will become obvious in the next paragraphs.

Following Hadwiger [46], we say that a point $p \in \mathbb{R}^{n} \backslash K$ illuminates a boundary point $b \in$ bd $K$, if the ray $\{p+\lambda(b-p): \lambda>0\}$ emanating from $p$ and passing through $b$ intersects the interior of $K$. Boltyanski [16] gave the following slightly 
different definition. A direction $u \in \mathbb{S}^{n-1}$ is said to illuminate $K$ at a boundary point $b \in$ bd $K$, if the ray $\{b+\lambda u: \lambda>0\}$ intersects the interior of $K$. It is easy to see that the minimum number of directions that illuminate each boundary point of $K$ is equal to the minimum number of points that illuminate each boundary point of $K$, which in turn is equal to the illumination number of $K$ (as defined in the paragraph above).

Gohberg and Markus [40] asked how large inf $\{N(K, \lambda K): 0<\lambda<1\}$ can be. It also follows easily that this number is equal to $i(K)$.

The following dual formulation of the definition of the illumination number was found independently by P. Soltan, V. Soltan [90] and by Bezdek [11]. First, recall that an exposed face of a convex body $K$ is the intersection of $K$ with a supporting hyperplane. Now, let $K$ be a convex body in $\mathbb{R}^{n}$ containing the origin in its interior. Then $i(K)$ is the minimum size of a family of hyperplanes in $\mathbb{R}^{n}$ such that each exposed face of the polar $K^{*}$ of $K$ is strictly separated from the origin by at least one of the hyperplanes in the family (for the definition of $K^{*}$, see the introduction).

Any smooth convex body (ie., a convex body with a unique support hyperplane at each boundary point) in $\mathbb{R}^{n}$ is illuminated by $n+1$ directions. Indeed, for a smooth convex body, the set of directions illuminating a given boundary point is an open hemisphere of $\mathbb{S}^{n-1}$, and one can find $n+1$ points (eg., the vertices of a regular simplex) in $\mathbb{S}^{n-1}$ with the property that every open hemisphere contains at least one of the points. Thus, these $n+1$ points in $\mathbb{S}^{n-1}$ (ie., directions) illuminate any smooth convex body in $\mathbb{R}^{n}$. It is easy to see that no convex body is illuminated by less than $n+1$ directions.

On the other hand, the illumination number of the cube is $2^{n}$, since no two vertices of the cube share an illumination direction. An important unsolved problem in Discrete Geometry is the Gohberg-Markus-Levi-Boltyanski-Hadwiger Conjecture (or, Illumination Conjecture), according to which for any convex body $K$ in $\mathbb{R}^{n}$, we have $i(K)=2^{n}$, where equality is attained only when $K$ is an affine image of the cube.

In this section, we mention some results on illumination. For a more complete account of the current state of the problem, see [8, 10, 21, 59, 95]. In Chap. VI. of [15], among many other facts on illumination, one can find a proof of the equivalence of the first four definitions of $i(K)$ given at the beginning of this section. Quantitative versions of the illumination number are discussed in the article of Bezdek and Khan in this volume [13]. Connections of the illumination number to other quantities are discussed in [102, 103].

One detail of the history of the conjecture may tell a lot about it. It was asked several times in different formulations (see the different definitions of $i(K)$ above), first in 1960 (though, Levi's study of $N(K$, int $K$ ) on the plane is from 1955). Several partial results appeared solving the conjecture for special families of convex bodies. Yet, the best general bound is an immediate consequence of Rogers' Theorem 3.1 (more precisely, Corollary 3.5) dating 1957 combined with the Rogers-Shepard inequality [84], according to which $\operatorname{vol}(K-K) \leq\left(\begin{array}{c}2 n \\ n\end{array}\right) \operatorname{vol}(K)$ for any convex body $K$ in $\mathbb{R}^{n}$. 
Theorem 6.1 (Rogers [80]) Let $K$ be a convex body in $\mathbb{R}^{n}$. Then

$$
i(K) \leq \begin{cases}2^{n}(n \ln n+n \ln \ln n+5 n) & \text { if } K=-K \\
\left(\begin{array}{c}
2 n \\
n
\end{array}\right)(n \ln n+n \ln \ln n+5 n) & \text { otherwise }\end{cases}
$$

By [56], the Illumination Conjecture holds on the plane. Papadoperakis [75] proved $i(K) \leq 16$ in dimension three. The upper bound in the conjecture (that is, not the equality case) was verified in the following cases: if $K=-K \subset \mathbb{R}^{3}$ (Lassak [54]), if $K \subset \mathbb{R}^{3}$ is a convex polyhedron with at least one non-trivial affine symmetry (Bezdek [11]), if $K \subset \mathbb{R}^{3}$ is symmetric about a plane (Dekster [23]).

\subsection{Borsuk's Problem and Illuminating Sets of Constant Width}

The problem of illumination is closely related to another classical question in geometry. Borsuk's problem [20] (or, Borsuk's Conjecture, though, he formulated it as a question) asks whether every bounded set $X$ in $\mathbb{R}^{n}$ can be partitioned into $n+1$ sets of diameter less than the diameter of $X$ (cf. [60] for a comprehensive survey). The minimum number of such parts is the Borsuk number of $X$, and clearly, it is at most the illumination number of $\operatorname{conv}(X)$. Since any bounded set in $\mathbb{R}^{n}$ is contained in a set of constant width of the same diameter, it follows that any upper bound on the illumination number of sets of constant width in a certain dimension is also a bound on the maximum Borsuk number in the same dimension.

The affirmative answer to Borsuk's problem in the plane was proved by Borsuk, then, in three-space by Perkal [76] and Eggleston [26] (in the case of finite, threedimensional sets, see Grünbaum [45], Heppes-Révész [48] and Heppes [47]). It was first shown by Lassak [53] (see also [14, 101]) that sets of constant width in $\mathbb{R}^{3}$ can be illuminated by three pairs of opposite directions. It would be a nice alternative proof of the bound 4 on the Borsuk number in three-space, if one could show that threedimensional sets of constant width have illumination number 4 (see Conjecture 3.3.5. in [8]).

In 1993 by an ingenious proof, Kahn and Kalai [52] (based on a deep combinatorial result of Frankl and Wilson [37]) showed that if $n$ is large enough, then there is a finite set in $\mathbb{R}^{n}$ whose Borsuk number is greater than $(1.2)^{\sqrt{n}}$, thus answering Borsuk's question in the negative. That result made the following bound on the illumination number by Schramm [87] all the more relevant. Currently, this is also the best general bound for the Borsuk number.

Theorem 6.2 (Schramm [87]) In any dimension $n$ for any set $W$ of constant width in $\mathbb{R}^{n}$, we have

$$
i(W) \leq 5 n \sqrt{n}(4+\ln n)\left(\frac{3}{2}\right)^{n / 2} .
$$


By a fine analysis of Schramm's method, Bezdek (Theorem 6.8.3. of [8]) extended Theorem 6.2 to the class of those convex bodies $W$ that can be obtained as $W=$ $\cap_{x \in X}\left(x+\mathbf{B}_{2}^{n}\right)$ for some $X \subset \mathbb{R}^{n}$ compact set with diam $X \leq 1$. Note that a set $W$ is of constant width one if and only if, $W=\cap_{x \in W}\left(x+\mathbf{B}_{2}^{n}\right)$.

We sketch the proof. First, we give yet another way to compute the illumination number of a convex body $K$. Let $b$ be a boundary point of $K$, and consider its Gauss image $\beta(b) \subset \mathbb{S}^{n-1}$ consisting of the inner unit normal vectors of all hyperplanes supporting $K$ at $b$. It is a closed, spherically convex set. We denote the open polar of a subset of the sphere $F \subset \mathbb{S}^{n-1}$ by $F^{+}=\left\{u \in \mathbb{S}^{n-1}:<u, f>>\right.$ 0 for all $f \in F\}$. Consider the set family $\mathcal{F}=\left\{(\beta(b))^{+}: b \in\right.$ bd $\left.K\right\}$. Clearly, the directions $u_{1}, \ldots, u_{m} \in \mathbb{S}^{n-1}$ illuminate $K$ if and only if, each member of $\mathcal{F}$ contains at least one $u_{i}$. In other words, we are looking for a small cardinality transversal to the set family $\mathcal{F}$ (for definitions, see Sect.5.1). We note that the idea of considering the Gauss image and $\mathcal{F}$ to bound the illumination number also appears in $[9,11,14]$.

Now, consider a set $W=\bigcap_{x \in X}\left(x+\mathbf{B}_{2}^{n}\right)$ with a compact set $X \subset \mathbb{R}^{n}$ of diameter at most one. To make the problem of bounding $i(W)$ finite, we take a covering of $\mathbb{S}^{n-1}$ by spherical caps of Euclidean diameter $\varepsilon:=\sqrt{\frac{2 n}{2 n-1}}-1$, say $C_{1} \cup \ldots \cup C_{N}=\mathbb{S}^{n-1}$. Such covering exists with $N \leq\left(1+\frac{4}{\varepsilon}\right)^{n}$ by the simple bound (6). We could use a better bound, but that would not yield any visible improvement on the bound on $i(W)$. Let

$$
U_{i}:=\bigcup_{\beta(b) \cap C_{i} \neq \emptyset} \beta(b),
$$

and consider the set family $\mathcal{G}=\left\{U_{i}^{+}: i=1, \ldots, N\right\}$. Clearly, any transversal to the finite set family $\mathcal{G}$ is a transversal to $\mathcal{F}$, and hence, is a set that illuminates $K$. One can show that

$$
\operatorname{diam}\left(U_{i}\right) \leq 1+\varepsilon
$$

Let $V(t):=\inf \left\{\sigma\left(F^{+}\right): F \subset \mathbb{S}^{n-1}, \operatorname{diam} S \leq t\right\}$. A key element of the proof is the highly non-trivial claim that

$$
V(t) \geq \frac{1}{\sqrt{8 \pi n}}\left(\frac{3}{2}+\frac{\left(2-\frac{1}{n}\right) t^{2}-2}{4-\left(2-\frac{2}{n}\right) t^{2}}\right)^{-\frac{n-1}{2}}
$$

for all $0<t<\sqrt{\frac{2 n}{n-1}}$ and $n \geq 3$.

We notice that by $(16), \frac{\sigma}{V(1+\varepsilon)}$ is a fractional transversal to $\mathcal{G}$. Now, the original proof is completed by applying the dual of (10) to get $i(W) \leq\left\lfloor 1+\frac{\ln N}{-\ln (1-V(1+\varepsilon))}\right\rfloor$. Substituting the bound on $N$ and (17), the theorem follows. Another way to complete the proof is to use the dual of Lemma 5.2, which yields the slightly worse bound $i(W)<\frac{1+\ln N}{V(1+\varepsilon)}$. 


\subsection{Fractional Illumination}

The notion of fractional illumination was defined in [65], and then further studied in [4].

Definition 6.3 The fractional illumination number of a convex body $K$ in $\mathbb{R}^{n}$ is

$$
i^{*}(K)=N^{*}(K, \text { int } K) .
$$

It was observed in [65] that by (12) and the Rogers-Shepard inequality ( $\operatorname{vol}(K-$ $\left.K) \leq\left(\begin{array}{c}2 n \\ n\end{array}\right) \operatorname{vol}(K)\right)$ we have

$$
i^{*}(K) \leq \begin{cases}2^{n} & \text { if } K=-K \\
\left(\begin{array}{c}
2 n \\
n
\end{array}\right) & \text { otherwise }\end{cases}
$$

The fractional form of the Illumination Conjecture (weaker than the original) reads: $i^{*}(K) \leq 2^{n}$, and equality is attained by parallelotopes only. When $K$ is symmetric, the case of equality was settled by Artstein-Avidan and Slomka [5] using a lemma by Schneider.

Interestingly, no better bound is known, so the fractional form of the Illumination Conjecture does not seem much easier than the original. On the other hand, just as in general, for $N(K, L)$ and $N^{*}(K, L)$, we do not have an upper bound of $i(K)$ in terms of $i^{*}(K)$.

The fractional version of Borsuk's problem can be stated in a natural way, and was investigated in [49] using the language of multiple Borsuk coverings. We note that the example of a set in $\mathbb{R}^{n}$ with high Borsuk number given by Kahn and Kalai (see Sect. 6.1) is a set with high fractional Borsuk number as well.

\section{Decomposability of Multiple Coverings}

An $m$-fold covering of $\mathbb{R}^{n}$ by translates of a set $K$ is a family $\mathcal{F}$ of translates of $K$ such that each point is contained in at least $m$ members. It is a natural question whether, for a particular $K$, if $m$ is large enough (say, at least $m(K)$ ), then all $m$-fold coverings of $\mathbb{R}^{n}$ by translates of $K$ can be decomposed into two coverings. That is, can $\mathcal{F}$ be colored with two colors such that each color class of $\mathcal{F}$ is a covering of $\mathbb{R}^{n}$ ?

It was proved in [66] that if $K$ is a centrally symmetric convex polygon then such $m(K)$ exists. This was generalized to all convex polygons in [74, 97].

Arguably the most natural special case was asked by Pach [67]: consider the open unit disk. The un-published manuscript [58] was cited several times as having given a positive answer in this case, though, Pach [71] warned that the result "has not been independently verified." The following result of Mani-Levitska and Pach (see [1]) 
also suggested that such $m(K)$ should exist for unit disks. For every $n \geq 2$, there is a positive constant $c_{n}$ with the following property. For every positive integer $m$, any $m$-fold covering of $\mathbb{R}^{n}$ with unit balls can be decomposed into two coverings, provided that no point of the space belongs to more than $c_{n} 2^{m / n}$ balls. This result was one of the first geometric applications of the Lovász local lemma.

Pach and Pálvölgyi [69] recently showed that, very surprisingly, there is no such $m(K)$ for the open unit disk.

Their proof consists of a combinatorial part followed by an intricate geometric argument. First, based on [73], they construct a finite abstract hypergraph, with a non-decomposable multiple covering. Then, the hypergraph is given a geometric realization, that is, the vertex set is mapped to a set of points on the plane, and the edges are mapped to open unit disks in an incidence-preserving manner. Finally, this $m$-fold covering by disks of this finite planar set is extended to an $m$-fold covering of the whole plane without adding any disk that contains any of the points in the finite set.

For more on decomposability of coverings, see [70], and the more recent paper [69].

\section{An Asymptotic View}

In this section, we present two topics to illustrate the point of view taken in the asymptotic theory of convex bodies on the problem of translative coverings.

\subsection{Sudakov's Inequality}

Sudakov's Inequality relates the minimum number of Euclidean balls that cover a symmetric convex body to the mean width of the body, where the latter is defined as

$$
w(K)=\int_{\mathbb{S}^{n-1}} h_{K}(u)+h_{K}(-u) \mathrm{d} \sigma(u) .
$$

(See the definition of $\sigma$ and $h_{K}$ in the introduction.)

Theorem 8.1 (Sudakov's inequality, [93]) For any symmetric convex body $K$ in $\mathbb{R}^{n}$ and any $t>0$, we have

$$
\log N\left(K, t \mathbf{B}_{2}^{n}\right) \leq c n\left(\frac{w(K)}{t}\right)^{2}
$$

with an absolute constant $c>0$. 
It was observed by Tomczak-Jaegermann [99], that this inequality can be obtained from a dual form proved by Pajor and Tomczak-Jaegermann [72].

Theorem 8.2 (Dual Sudakov inequality) For any symmetric convex body $K$ in $\mathbb{R}^{n}$ and any $t>0$, we have

$$
\log N\left(\mathbf{B}_{2}^{n}, t K\right) \leq c n\left(\frac{w\left(K^{*}\right)}{t}\right)^{2}
$$

with an absolute constant $c>0$.

First, we sketch a proof of Theorem 8.2 due to Talagrand [96], [55], and later turn to the proof of Theorem 8.1. The main idea is to apply a volumetric argument, but, instead of using the Lebesgue measure, one uses the Gaussian measure. Recall, that the Gaussian measure $\gamma_{n}$ is an absolutely continuous probability measure on $\mathbb{R}^{n}$, with density

$$
\mathrm{d} \gamma_{n}(x)=\frac{e^{-|x|^{2} / 2}}{(2 \pi)^{n / 2}} \mathrm{~d} x
$$

First, by computation one obtains that for any origin-symmetric convex body $K$ in $\mathbb{R}^{n}$ and any translation vector $z \in \mathbb{R}^{n}$, we have

$$
\gamma_{n}(K+z) \geq e^{-|z|^{2} / 2} \gamma_{n}(K)
$$

Next, we consider a maximal set $\left\{x_{1}, \ldots, x_{N}\right\}$ in $\mathbf{B}_{2}^{n}$ with the property that $\| x_{i}-$ $x_{j} \|_{K} \geq t$ for all $i, j$ pairs. Now, for any rescaling factor $\lambda>0$, we have that $\left\{\lambda x_{i}+\right.$ $\left.\frac{\lambda t}{2} K: i=1, \ldots, N\right\}$ is a packing in $\lambda \mathbf{B}_{2}^{n}$, and thus, the total $\gamma_{n}$-measure of these sets is at most one. Integration in polar coordinates yields that

$$
\gamma_{n}\left(\frac{\lambda t}{2} K\right) \geq 1-\frac{2 c \sqrt{n}}{\lambda t} w\left(K^{*}\right)
$$

for an absolute constant $c>0$. With the choice $\lambda=4 c \sqrt{n} w\left(K^{*}\right) / t$, we have $\gamma_{n}(K) \geq \frac{1}{2}$. Finally, using (20), we obtain the bound in Theorem 8.2.

We note that this proof yields a little more than stated in the Theorem. We obtain an upper bound on the minimum size of a covering of $\mathbf{B}_{2}^{n}$ by translates of $t K$ with the constraint that the translation vectors are in $\mathbf{B}_{2}^{n}$.

The following Lemma is the key to reducing Theorem 8.1 to Theorem 8.2.

Lemma 8.3 (Tomczak-Jaegermann [99]) For any origin-symmetric convex body $K$ in $\mathbb{R}^{n}$, and any $t>0$, we have

$$
N\left(K, t \mathbf{B}_{2}^{n}\right) \leq N\left(K, 2 t \mathbf{B}_{2}^{n}\right) N\left(\mathbf{B}_{2}^{n}, \frac{t}{8} K^{*}\right)
$$


Proof of Lemma 8.3 Observe that $2 K \cap\left(\frac{t^{2}}{2} K^{*}\right) \subseteq t \mathbf{B}_{2}^{n}$. Thus, by (4),

$$
\begin{aligned}
N\left(K, t \mathbf{B}_{2}^{n}\right) \leq & N\left(K, 2 K \cap \frac{t^{2}}{2} K^{*}\right) \leq N\left(K, \frac{t^{2}}{4} K^{*}\right) \leq \\
& N\left(K, 2 t \mathbf{B}_{2}^{n}\right) N\left(\mathbf{B}_{2}^{n}, \frac{t}{8} K^{*}\right) .
\end{aligned}
$$

Proof of Theorem 8.1 Combining Lemma 8.3 and Theorem 8.2, we have

$$
t^{2} \log N\left(K, t \mathbf{B}_{2}^{n}\right) \leq \frac{1}{4}(2 t)^{2} \log N\left(K, 2 t \mathbf{B}_{2}^{n}\right)+64(t / 8)^{2} \log N\left(\mathbf{B}_{2}^{n}, \frac{t}{8} K^{*}\right)
$$

Taking supremum over all $t>0$, we get

$$
\frac{3}{4} \sup _{t>0}\left\{t^{2} \log N\left(K, t \mathbf{B}_{2}^{n}\right)\right\} \leq 64 \sup _{t>0}\left\{t^{2} \log N\left(\mathbf{B}_{2}^{n}, t K^{*}\right)\right\} \leq 64 c n(w(K))^{2} .
$$

\subsection{Duality of Covering Numbers}

We briefly mention the following open problem in geometric analysis, for a comprehensive discussion, cf. Chap. 4 of [3].

Conjecture 8.4 There are universal constants $c, C>0$ such that for any dimension $n$ and any two symmetric convex bodies $K$ and $L$ in $\mathbb{R}^{n}$, we have

$$
N(K, L) \leq N\left(L^{*}, c K^{*}\right)^{C}
$$

The problem is known as the Duality of entropy, and was posed by Pietsch [77]. An important special case, when $K$ or $L$ is a Euclidean ball (or, equivalently, an ellipsoid) was confirmed by Artstein-Avidan, Milman and Szarek [2].

\section{Covering by Sequences of Sets}

So far, we considered problems where a set was to be covered by translates of another fixed set. Now, we turn to problems where a family $\mathcal{F}$ of sets is given, and we need to find a translation for each set in $\mathcal{F}$ to obtain a covering of a given set $C$. If such translations exist, we say that $\mathcal{F}$ permits a translative covering of $C$. We call $\mathcal{F}$ a bounded family, if the set of diameters of members of $\mathcal{F}$ is a bounded set.

For a comprehensive account of coverings by sequences of convex sets, see the surveys $[32,43]$. 


\subsection{Covering (Almost) the Whole Space.}

Clearly, for $\mathcal{F}$ to permit a translative covering of $\mathbb{R}^{n}$, it is necessary that the total volume of the members of $\mathcal{F}$ be infinite. It is not sufficient, though. Indeed, consider rectangles of side lengths $i$ by $1 / i^{2}$ for $i=1,2, \ldots$ on the plane. Their total area is infinite, and yet, according to Bang's theorem, they do not permit a translative covering of $\mathbb{R}^{2}$ [32]. On the other hand, if a family of planar convex sets is bounded and has infinite total area, then it permits a translative covering of $\mathbb{R}^{2}[42,51]$. It is an open problem whether the same holds for $n>2$.

A covering of almost all of some set $C$ is a covering of a subset of $C$ whose complement in $C$ is of measure zero.

Theorem 9.1 (Groemer, [44]) Let $\mathcal{F}$ be a bounded family of Lebesgue measurable sets. Then $\mathcal{F}$ permits a translative covering of almost all of $\mathbb{R}^{n}$ if and only if, $\sum_{F \in \mathcal{F}} \operatorname{vol}(F)=\infty$.

Indeed, let $\mathcal{F}=\left\{F_{1}, F_{2}, \ldots\right\}$ be a bounded family with infinite total volume. Clearly, it is sufficient to cover almost all of the cube $C=[-1 / 2,1 / 2]^{n}$. We may assume that $F \subset C$ for all $F \in \mathcal{F}$.

We find the translation vectors inductively. Let $x_{1}=0$. If $x_{k}$ is defined, we denote the uncovered part by $E_{k}=C \backslash\left(\bigcup_{j=1}^{k}\left(F_{j}+x_{j}\right)\right)$. We choose $x_{k+1}$ in such a way that

$$
\frac{\operatorname{vol}\left(\left(F_{k+1}+x_{k+1}\right) \cap E_{k}\right)}{\operatorname{vol}\left(F_{k+1}\right)} \geq \frac{1}{2^{n}} \operatorname{vol}\left(E_{k}\right) \text {. }
$$

It is possible, since

$$
\begin{aligned}
& \frac{1}{2^{n}} \int_{2 C} \operatorname{vol}\left(\left(F_{k+1}+x\right) \cap E_{k}\right) \mathrm{d} x=\frac{1}{2^{n}} \int_{2 C} \int_{C} \chi_{F_{k+1}}(y-x) \chi_{E_{k}}(y) \mathrm{d} y \mathrm{~d} x \\
& \quad=\frac{1}{2^{n}} \int_{C} \chi_{E_{k}}(y) \int_{2 C} \chi_{F_{k+1}}(y-x) \mathrm{d} x \mathrm{~d} y=\frac{1}{2^{n}} \operatorname{vol}\left(F_{k+1}\right) \operatorname{vol}\left(E_{k}\right) .
\end{aligned}
$$

It is easy to see that (21) implies that $\lim _{k \rightarrow \infty} \operatorname{vol}\left(E_{k}\right)=0$.

We note that the condition that $\mathcal{F}$ is bounded may be replaced by the condition that $\mathcal{F}$ contains only convex sets, see [43].

\subsection{A Sufficient Condition for a Family of Homothets}

For convex bodies $K$ and $L$, we define $f(K, L)$ as the infimum of those $t>0$, such that for any family $\mathcal{F}$ of homothets of $L$ with coefficients $0<\lambda_{1}, \lambda_{2}, \cdots<1$, the following holds: 
If $\sum_{i} \lambda_{i}^{d} \geq t$ then $\mathcal{F}$ permits a translative covering of $K$.

We set $f(n):=\sup \left\{f(K, K): K \subset \mathbb{R}^{n}\right.$ a convex body $\}$.

The question of bounding $f(2)$ was originally posed by L. Fejes Tóth [33] (cf. Sect. 3.2 in [21]). He conjectured that $f(2) \leq 3$. Januszewski [50] showed that $f(2) \leq 6.5$. In higher dimensions Meir and Moser [62], and later, A. Bezdek and K. Bezdek [12] considered the cube and proved that $f\left([0,1]^{d}\right)=2^{d}-1$. Using a simple argument based on saturated packings by half-sized copies (see (6)), the author [64] showed

$$
f(K, L) \leq 2^{n} \frac{\operatorname{vol}\left(K+\frac{L \cap(-L)}{2}\right)}{\operatorname{vol}(L \cap(-L))},
$$

from which the bound

$$
f(K, K) \leq\left\{\begin{array}{l}
3^{n}, \text { if } K=-K \\
6^{n}, \text { in general. }
\end{array}\right.
$$

follows.

On the other hand, clearly, $f(K, K) \geq n$ since we may consider $n$ homothetic copies of $K$ with homothety ratios slightly below one, and use the lower bound on the illumination number of $K$ (see Sect.6).

\subsection{A Necessary Condition for a Family of Homothets}

A converse to the problem discussed above was formulated by V. Soltan [89] (cf. Sect. 3.2 in [21]). Let

$$
g(K):=\inf \left\{\sum_{i} \lambda_{i}: K \subseteq \bigcup_{i} \lambda_{i} K+x_{i}, 0<\lambda_{i}<1\right\},
$$

and $g(n):=\inf \left\{g(K): K \subset \mathbb{R}^{n}\right.$ a convex body $\}$. V. Soltan conjectured $g(n) \geq n$.

Since the $n$-dimensional simplex $\Delta$ can be covered by $n+1$ translates of $\frac{n}{n+1} \Delta$, we have that $g(n) \leq g(\Delta) \leq n$. V. Soltan and É. Vásárhelyi [88] showed $g(2) \geq 2$, and also proved that the conjecture holds when only $n+1$ homothets are allowed.

Soltan's conjecture was confirmed in an asymptotic sense in [64]: $\lim _{n \rightarrow \infty} \frac{g(n)}{n}=1$.

Acknowledgements The author is grateful for the many illuminating conversations with Gábor Fejes Tóth about covering problems in general, and about this manuscript. 


\section{References}

1. N. Alon, J.H. Spencer, The Probabilistic Method. With an Appendix on the Life and Work of Paul Erdôs, 3rd ed. (Hoboken, Wiley, 2008) (English)

2. S. Artstein, V. Milman, S.J. Szarek, Duality of metric entropy. Ann. Math. (2) 159(3), 13131328 (2004). MR2113023 (2005h:47037)

3. S. Artstein-Avidan, A. Giannopoulos, Mathematical surveys and monographs, Asymptotic Geometric Analysis. Part I (American Mathematical Society, Providence, 2015). MR3331351

4. S. Artstein-Avidan, O. Raz, Weighted covering numbers of convex sets. Adv. Math. 227(1), 730-744 (2011)

5. S. Artstein-Avidan, B.A. Slomka, On Weighted Covering Numbers and the LeviHadwiger Conjecture. Israel Journal of Mathematics 209(1), 125-155 (September 2015), arXiv: 1310.7892

6. K. Ball, Volume ratios and a reverse isoperimetric inequality. J. Lond. Math. Soc. (2) 44(2), 351-359 (1991). MR1136445 (92j:52013)

7. K. Ball, Volumes of sections of cubes and related problems, Geometric Aspects of Functional Analysis (1987-88) (1989), pp. 251-260. MR1008726 (90i:52019)

8. K. Bezdek, Classical Topics in Discrete Geometry, CMS Books in Mathematics/Ouvrages de Mathématiques de la SMC (Springer, New York, 2010)

9. K. Bezdek, Gy Kiss, On the X-ray number of almost smooth convex bodies and of convex bodies of constant width. Can. Math. Bull. 52(3), 342-348 (2009). MR2547800 (2010m:52009)

10. K. Bezdek, The illumination conjecture and its extensions. Period. Math. Hung. 53(1-2), 59-69 (2006)

11. K. Bezdek, The problem of illumination of the boundary of a convex body by affine subspaces. Mathematika 38(2), 362-375 (1991, 1992). MR1147835 (92m:52020)

12. A. Bezdek, K. Bezdek, Eine hinreichende Bedingung für die Überdeckung des Einheitswürfels durch homothetische Exemplare im n- dimensionalen euklidischen Raum, Beiträge Algebra Geom. 17 (1984), pp. 5-21. MR755762 (85h:52017)

13. K. Bezdek, M.A. Khan, The geometry of homothetic covering and illumination, in Discrete geometry and symmetry (to appear), 2018

14. K. Bezdek, Z. Lángi, M. Naszódi, P. Papez, Ball-polyhedra. Discret. Comput. Geom. 38(2), 201-230 (2007). MR2343304 (2008i:52001)

15. V. Boltyanski, H. Martini, P.S. Soltan, Excursions into Combinatorial Geometry (Universitext, Springer, Berlin, 1997). MR1439963 (98b:52001)

16. V. Boltyanski, The problem of illuminating the boundary of a convex body. Izv. Mold. Fil. AN SSSR 76, 77-84 (1960)

17. T. Bonnesen, W. Fenchel, Theory of convex bodies. Transl. from the German and ed. by L. Boron, C. Christenson, B. Smith, with the Collaboration of W. Fenchel (Moscow, Idaho, USA): BCS Associates. IX, 172 p. (1987) (English)

18. K. Böröczky Jr., Finite Packing and Covering, Cambridge Tracts in Mathematics, vol 154 (Cambridge University Press, Cambridge, 2004). MR2078625 (2005g:52045)

19. K. Böröczky Jr., K. Böröczky Jr., Covering the spere by equal spherical balls, Discrete and Computational Geometry (Springer, Berlin, 2003), pp. 235-251

20. K. Borsuk, Drei sätze über die n-dimensionale euklidische sphäre, Fundamenta Mathematicae 20 (1933), no. 1, 177-190 (ger)

21. P. Brass, W. Moser, J. Pach, Research Problems in Discrete Geometry (Springer, New York, 2005)

22. H.S.M. Coxeter, L. Few, C.A. Rogers, Covering space with equal spheres. Mathematika 6, 147-157 (1959). MR0124821 (23 \#A2131)

23. B.V. Dekster, Each convex body in $E^{3}$ symmetric about a plane can be illuminated by 8 directions. J. Geom. 69(1-2), 37-50 (2000). MR1800455 (2001m:52003)

24. I. Dumer, Covering spheres with spheres, Discrete Comput. Geom. 38(4), 665Â-679 (2007)

25. I. Dumer, Covering spheres with spheres (2018), arXiv:0606002v2 [math] 
26. H.G. Eggleston, Covering a three-dimensional set with sets of smaller diameter. J. Lond. Math. Soc. 30, 11-24 (1955). MR0067473 (16,734b)

27. P. Erdôs and L. Lovász, Problems and results on 3-chromatic hypergraphs and some related questions, Infinite and finite sets (Colloquium, Keszthely, 1973; dedicated to P. Erdős on his 60th birthday), Vol. II, 1975, pp. 609-627. Colloquium Mathematical Society, János Bolyai, vol. 10. MR0382050 (52 \#2938)

28. P. Erdős, C.A. Rogers, Covering space with convex bodies. Acta Arith. 7, 281-285 $(1961 / 1962)$

29. G. Fejes Tóth, A note on covering by convex bodies. Can. Math. Bull. 52(3), 361-365 (2009)

30. L. Fejes Tóth, Lagerungen in der Ebene, auf der Kugel und im Raum, Die Grundlehren der Mathematischen Wissenschaften in Einzeldarstellungen mit besonderer Berücksichtigung der Anwendungsgebiete, (Band LXV, Springer, Berlin, 1953). MR0057566 (15,248b)

31. G. Fejes Tóth, New results in the theory of packing and covering, in Convexity and its Applications, Collect. Surv. (1983), 318-359; 1983 (English)

32. G. Fejes Tóth, Packing and covering, Handbook of Discrete and Computational Geometry, 2nd ed. (2004), pp. 25-53 (English)

33. L. Fejes Tóth, Personal communication (1984)

34. G. Fejes Tóth, Recent progress on packing and covering, in Advances in Discrete and Computational Geometry: Proceedings of the 1996 AMSIMS-SIAM Joint Summer Research Conference on Discrete and Computational Geometry: Ten Years Later, South Hadley, USA, 14-18 July 1996 (1996), pp. 145-162 (English)

35. G. Fejes Tóth, W. Kuperberg, A survey of recent results in the theory of packing and covering, New Trends in Discrete and Computational Geometry (1993), pp. 251-279. (English)

36. G. Fejes Tóth, W. Kuperberg, Packing and covering with convex sets, in Handbook of Convex Geometry, vol. B (1993), pp. 799-860 (English)

37. P. Frankl, R.M. Wilson, Intersection theorems with geometric consequences. Combinatorica 1(4), 357-368 (1981). MR647986 (84g:05085)

38. Z. Füredi, Matchings and covers in hypergraphs. Graphs Comb. 4(2), 115-206 (1988)

39. Z. Füredi, J.-H. Kang, Covering the n-space by convex bodies and its chromatic number. Discret. Math. 308(19), 4495-4500 (2008)

40. I. Gohberg, A. Markus, A problem on covering of convex figures by similar figures. Izv. Mold. Fil. AN SSSR 10, 87-90 (1960)

41. P. Gritzmann, Lattice covering of space with symmetric convex bodies. Mathematika 32(2), 311-315 (1985); (1986). MR834499

42. H. Groemer, Covering and packing properties of bounded sequences of convex sets. Mathematika 29, 18-31 (1982). (English)

43. H. Groemer, Coverings and packings by sequences of convex sets, Discrete Geometry and Convexity (New York, 1982); (1985), pp. 262-278

44. H. Groemer, Space coverings by translates of convex sets. Pac. J. Math. 82, 379-386 (1979). (English)

45. B. Grünbaum, A simple proof of Borsuk's conjecture in three dimensions. Math. Proc. Camb. Philos. Soc. 53, 776-778 (1957). MR0090072 (19,763d)

46. H. Hadwiger, Ungelöste probleme, nr. 38. Elem. Math. 15, 130-131 (1960)

47. A. Heppes, On the partitioning of three-dimensional point-sets into sets of smaller diameter. Magyar Tud. Akad. Mat. Fiz. Oszt. Közl. 7, 413-416 (1957). MR0095450 (20 \#1952)

48. A. Heppes, P. Révész, A splitting problem of Borsuk, Mat. Lapok 7 (1956), 108-111. MR0098353 (20 \#4814)

49. M. Hujter, Z. Lángi, On the multiple Borsuk numbers of sets. Israel J. Math. 199(1), 219-239 (2014). MR3219534

50. J. Januszewski, Translative covering a convex body by its homothetic copies. Stud. Sci. Math. Hung. 40(3), 341-348 (2003). MR2036964 (2005b:52044)

51. E.jr Makai, J. Pach, Controlling function classes and covering Euclidean space. Stud. Sci. Math. Hung. 18, 435-459 (1983). (English) 
52. J. Kahn, G. Kalai, A counterexample to Borsuk's conjecture. Bull. Amer. Math. Soc. (N.S.) 29(1), 60-62 (1993). MR1193538 (94a:52007)

53. M. Lassak, Illumination of three-dimensional convex bodies of constant width, in Proceedings of the 4th International Congress of Geometry: Thessaloniki, 1996 (1997), pp. 246-250. MR1470984 (98g:52013)

54. M. Lassak, Solution of Hadwiger's covering problem for centrally symmetric convex bodies in $E^{3}$, J. Lond. Math. Soc. (2) 30(3), 501-511 (1984). MR810959 (87e:52024)

55. M. Ledoux, M. Talagrand, Probability in Banach Spaces, Ergebnisse der Mathematik und ihrer Grenzgebiete (3) [Results in Mathematics and Related Areas (3)], vol. 23 (Springer, Berlin, 1991). Isoperimetry and Processes. MR1102015 (93c:60001)

56. F.W. Levi, Überdeckung eines Eibereiches durch Parallelverschiebung seines offenen Kerns. Arch. Math. (Basel) 6, 369-370 (1955). MR0076368 (17,888b)

57. L. Lovász, On the ratio of optimal integral and fractional covers. Discret. Math. 13(4), 383-390 (1975)

58. P. Mani-Levitska, J. Pach, Decomposition Problems for Multiple Coverings with Unit Balls (1986). Manuscript: Parts of the manuscript available at http://www.math.nyu.edu/ pach/ publications/unsplittable.pdf

59. H. Martini, V. Soltan, Combinatorial problems on the illumination of convex bodies. Aequ. Math. 57(2-3), 121-152 (1999)

60. J. Math. Sci. Around Borsuk's hypothesis. 154(4), 604-623 (2008). (English)

61. J. Matoušek, Lectures on discrete geometry (Graduate Texts in Mathematics), vol. 212 (Springer, New York, 2002)

62. A. Meir, L. Moser, On packing of squares and cubes. J. Comb. Theory 5, 126-134 (1968). MR0229142 (37 \#4716)

63. H. Minkowski, Allgemeine Lehrsätze über die convexen Polyeder. Nachr. Ges. Wiss. Göttingen, Math.-Phys. Kl. 1897, 198-219 (1897) (German)

64. M. Naszódi, Covering a set with homothets of a convex body. Positivity 14(1), 69-74 (2010). MR2596464

65. M. Naszódi, Fractional illumination of convex bodies. Contrib. Discret. Math. 4(2), 83-88 (2009)

66. J. Pach, Covering the plane with convex polygons. Discret. Comput. Geom. 1, 73-81 (1986). (English)

67. J. Pach, Decomposition of multiple packing and covering. Diskrete Geometrie, 2. Kolloq., Inst. Math. Univ. Salzburg 1980, 169-178 (1980). 1980 (English)

68. J. Pach, P.K. Agarwal, Combinatorial Geometry (New York, Wiley, 1995) (English)

69. J. Pach, D. Pálvölgyi, Unsplittable coverings in the plane. Adv. Math. 302, 433-457 (2016)

70. J. Pach, D. Pálvölgyi, G. Tóth, Survey on decomposition of multiple coverings, in GeometryIntuitive, Discrete, and Convex (2013), pp. 219-257. MR3204561

71. J. Pach, G. Tóth, Decomposition of multiple coverings into many parts. Comput. Geom. 42(2), 127-133 (2009). (English)

72. A. Pajor, N. Tomczak-Jaegermann, Remarques sur les nombres d'entropie d'un opérateur et de son transposé, C. R. Acad. Sci. Paris Sér. I Math. 301(15), 743-746 (1985). MR817602 (87f:47027)

73. D. Pálvölgyi, Indecomposable coverings with concave polygons. Discret. Comput. Geom. 44(3), 577-588 (2010). (English)

74. D. Pálvölgyi, G. Tóth, Convex polygons are cover-decomposable. Discret. Comput. Geom. 43(3), 483-496 (2010). (English)

75. I. Papadoperakis, An estimate for the problem of illumination of the boundary of a convex body in $E^{3}$, Geom. Dedicata 75(3), 275-285 (1999). MR1689273 (2000g:52014)

76. J. Perkal, Sur la subdivision des ensembles en parties de diamétre intérieure. Colloq. Math. 1, 45 (1947)

77. A. Pietsch, Theorie der Operatorenideale (Zusammenfassung), Friedrich-SchillerUniversität, Jena, 1972. Wissenschaftliche Beiträge der Friedrich-Schiller-Universität Jena. MR0361822 (50 \#14267) 
78. Proc. Amer. Math. Soc. On some covering problems in geometry. 144(8), 3555-3562 (2016). MR3503722

79. J. Radon, Über eine Erweiterung des Begriffes der konvexen Funktionen mit einer Anwendung auf die Theorie der konvexen Körper. Wien. Ber. 125, 241-258 (1916). (German)

80. C.A. Rogers, A note on coverings. Mathematika 4, 1-6 (1957)

81. C.A. Rogers, Covering a sphere with spheres. Mathematika 10, 157-164 (1963)

82. C.A. Rogers, Lattice coverings of space. Mathematika 6, 33-39 (1959)

83. C.A. Rogers, Packing and Covering, Cambridge Tracts in Mathematics and Mathematical Physics, vol. 54 (Cambridge University Press, New York, 1964)

84. C.A. Rogers, G.C. Shephard, The difference body of a convex body. Arch. Math. (Basel) 8, 220-233 (1957)

85. C.A. Rogers, C. Zong, Covering convex bodies by translates of convex bodies. Mathematika 44(1), 215-218 (1997)

86. W. Schmidt, Maßtheorie in der Geometrie der Zahlen. Acta Math. 102, 159-224 (1959). (German)

87. O. Schramm, Illuminating sets of constant width. Mathematika 35(2), 180-189 (1988)

88. V. Soltan, É. Vásárhelyi, Covering a convex body by smaller homothetic copies. Geom. Dedicata 45(1), 101-113 (1993). MR1199732 (94a:52040)

89. V. Soltan, Personal Communication (1990)

90. P.S. Soltan, V.P. Soltan, Illumination through convex bodies. Dokl. Akad. Nauk SSSR 286(1), 50-53 (1986). MR822098 (87f:52008)

91. J. Spencer, Asymptotic lower bounds for Ramsey functions. Discret. Math. 20(1), 69-76 (1977/78). MR0491337 (58\#10600)

92. S.K. Stein, Two combinatorial covering theorems. J. Comb. Theory Ser. A 16(3), 391-397 (1974)

93. V.N. Sudakov, Gaussian random processes, and measures of solid angles in Hilbert space, Dokl. Akad. Nauk SSSR 197, 43-45 (1971). MR0288832 (44 \#6027)

94. W. Süss, Über den Vektorenbereich eines Eikörpers. Jahresber. Dtsch. Math.-Ver. 37, 87-90 (1928). (German)

95. L. Szabó, Recent results on illumination problems, in Intuitive Geometry (Budapest, 1995) (1997), pp. 207-221. MR1470759 (98h:52015)

96. M. Talagrand, A new isoperimetric inequality for product measure and the tails of sums of independent random variables. Geom. Funct. Anal. 1(2), 211-223 (1991). MR1097260 (92j:60004)

97. G. Tardos, G. Toth, Multiple coverings of the plane with triangles. Discret. Comput. Geom. 38(2), 443-450 (2007). (English)

98. Th. Estermann, Über den Vektorenbereich eines konvexen Körpers. Math. Z. 28, 471-475 (1928). (German)

99. N. Tomczak-Jaegermann, Dualité des nombres d'entropie pour des opérateurs à valeurs dans un espace de Hilbert. C. R. Acad. Sci. Paris Sér. I Math. 305(7), 299-301 (1987). MR910364 (89c:47027)

100. J.-L. Verger-Gaugry, Covering a ball with smaller equal balls in $\mathbb{R}^{n}$. Discret. Comput. Geom. 33(1), 143-155 (2005). (English)

101. B. Weissbach, Invariante Beleuchtung konvexer Körper, Beiträge Algebr. Geom. 37(1), 9-15 (1996). MR1407801 (97j:52011)

102. C.M. Zong, Some remarks concerning kissing numbers, blocking numbers and covering numbers. Period. Math. Hung. 30(3), 233-238 (1995). MR1334968

103. C. Zong, The kissing number, blocking number and covering number of a convex body. Surv. Discret. Comput. Geom. 453, 529-548 (2008). MR2405694 